\title{
Landiolol Hydrochloride Ameliorates Liver Injury in a Rat Sepsis Model by Down Regulating Hepatic TNF- A
}

Yasuyo Yoshino, Subrina Jesmin, Majedul Islam, Nobutake Shimojo, Hideaki Sakuramoto, Masami Oki, Tanzila Khatun, Masato Suda, Satoru Kawano and Taro Mizutani*

Department of Emergency and Critical Care Medicine, University of Tsukuba, Tsukuba, Ibaraki, Japan

\begin{abstract}
Aims: The effects of a beta blocker, especially an ultra-short acting selective beta blocker, such as landiolol hydrochloride on the organ protection in sepsis are unclear. The present study aimed to investigate whether acute (early hours) liver injury in a rat model of sepsis induced by lipopolysaccharide (LPS) administration: a) can be corrected by administering landiolol and $b$ ) whether landiolol's effects on liver injury is accomplished by diminishing the elevated expression of inflammatory cytokine, such as tumor necrosis factor (TNF)- $\alpha$ and a vaso constrictor peptide, such as endothelin (ET)-1.

Methods: Eight (8)-week-old male Wistar rats were administered for three hours with either LPS ( $n=12)$, or continuously with LPS plus landiolol $(n=11)$. Control rats were treated with saline only in a similar manner as the treatment group during the relevant time points $(n=13)$.

Results: Following LPS administration, blood gas and hemodynamic parameters were significantly altered compared to control rats at $3 \mathrm{~h}$. Also, At $3 \mathrm{~h}$ after LPS administration, circulatory levels of ALT, AST, TNF- $\alpha$ and ET-1 were significantly increased. In addition, at $3 \mathrm{~h}$ after LPS administration significant features of hepatic injuries at morphological levels were also evident. Co-treatment of rats with LPS and landiolol ameliorated hepatic injury at 3 $\mathrm{h}$ post-treatment, as well as reversed elevated circulatory levels of factors associated with liver injury back to normal levels, such as AST and ALT, and local hepatic levels of TNF- $\alpha$.
\end{abstract}

Conclusion: Based on the current findings, it can be stated that landiolol may exert protective effects on liver injury in septic rats by normalizing local expression levels of inflammatory cytokine, such as TNF- $\alpha$.

Keywords: Landiolol hydrochloride; Liver injury; TNF- $\alpha$; Endothelin-1; Sepsis; Rat model

\section{Introduction}

Sepsis, a critical medical emergency, is associated with tissue hypoperfusion and metabolic impairment, which may contribute to the subsequent development of multiple organ failure normally associated with this disorder [1]. The liver is one of the organs that are normally damaged during the pathogenesis of sepsis and septic shock. Therefore, protecting the liver [2-4] is an important target in sepsis treatment and management. Although numerous studies on infection- or sepsisinduced liver injury have been conducted $[5,6]$, to date no effective treatment has been reported on this disorder. We know that during sepsis, liver functions are altered by the activation of inflammatory processes $[6,7]$ to date no effective treatment has been reported on this disorder. We know that during sepsis, liver functions are altered by the activation of inflammatory processes. For instance, both ex-vivo and in-vitro studies have demonstrated that tumor necrosis factor- $\alpha$ (TNF- $\alpha$ ) is released in response to lipopolysaccharide (LPS), primarily by Kupffer cells $[8,9]$. Specifically, LPS stimulates Kupffer cells to secrete TNF- $\alpha$, which, subsequently, contributes to the pathogenesis of LPS-induced liver injury by a direct or an indirect polymorphonuclear leucocyte-dependent mechanism $[9,10]$.

Endothelin-1 (ET-1), a potent vasoconstrictor with vasoproliferative activity, is believed to participate in the pathogenesis of sepsis, and its plasma (ET-1) levels significantly increase [11] in sepsis. The possible involvement of the ET system in human septic shock is further supported by a clear correlation between endothelin plasma levels and morbidity and mortality in septic patients $[12,13]$. Infusion of ET-1 in human causes cardiovascular changes, in part resembling those seen during sepsis i.e. decreased cardiac output and vasoconstriction in the pulmonary, renal and splanchnic circulation [13]. In our previous study, we clearly demonstrated that ET-1 is upregulated in liver during sepsis in a time-dependent manner [14].

Landiolol, an ultra-short-acting and highly cardio-selective $\beta$-1 blocker, has become useful for various medical problems in recent days, as evidenced from both clinical and animal studies. Recent studies have demonstrated that co-treatment of LPS with landiolol protects against acute lung injury and cardiac dysfunction in a rat model of LPS-induced systemic inflammation, which was also associated with a significant reduction in serum levels of the inflammation mediator HMGB-1 and histological lung damage [15]. More recently, our group has demonstrated that landiolol treatment significantly normalized various components of altered cardiac ET-1 signaling system in septic rat [16]. In addition, Ogura et al. reported the reno-protective effects of landiolol hydrochloride during sepsis by normalizing the altered expression of ET-1 and HIF-l alpha levels [17]. However, no study has

*Corresponding author: Taro Mizutani, Department of Emergency and Critical Care Medicine, University of Tsukuba, Tsukuba, Ibaraki- 305-8575, Japan, Tel: +81-29-8533210, 3081; Fax: +81-29-853-5984; E-mail: mizutani@md.tsukuba.ac.jp

Received January 24, 2015; Accepted March 14, 2015; Published March 16 , 2015

Citation: Yoshino Y, Jesmin S, Islam M, Shimojo N, Sakuramoto H, et al. (2015) Landiolol Hydrochloride Ameliorates Liver Injury in a Rat Sepsis Model by Down Regulating Hepatic TNF-A. J Vasc Med Surg 3: 194. doi:10.4172/23296925.1000194

Copyright: $\odot 2015$ Yoshino Y, et al. This is an open-access article distributed unde the terms of the Creative Commons Attribution License, which permits unrestricted use, distribution, and reproduction in any medium, provided the original author and source are credited. 
yet investigated whether landiolol has protective effects on liver injury during sepsis.

In the current study, we investigated whether landiolol hydrochloride plays an important role in ameliorating liver injury during sepsis and whether such blockage involves attenuation of hepatic TNF- $\alpha$ and ET-1 expression.

\section{Materials and Methods}

\section{Animal preparation}

In the present study, we used male Wistar rats $(200-250 \mathrm{~g}, 8$ weeks old). Sepsis was induced by intra-peritoneal (IP) administration of bacterial LPS from Escherichia coli 055: B5 (Sigma Aldrich, Saint Louis, USA) $(15 \mathrm{mg} / \mathrm{kg})$, dissolved in sterile saline. The rats were (n $=38$ ) randomized into group 1 (control, $n=13$ ), group 2 (LPS, $n=12$ ) and group 3 (LPS + landiolol hydrochloride, $n=11$ ). Group 1 received an equal volume of vehicle (sterile saline; $2 \mathrm{ml} /$ body), without LPS. LPS (15 mg/kg, intraperitoneal) was administered at time point $0 \mathrm{~h}$ in groups 2 and 3 , and then the rats were killed after $3 \mathrm{~h}$ post-treatment. However, for group 3, 15 min before LPS or vehicle administration, landiolol hydrochloride was administered continuously intravenously $(100 \mu \mathrm{g} / \mathrm{kg} / \mathrm{min})$. This dosage and frequency of landiolol $(100 \mathrm{mg} / \mathrm{kg} /$ min) from our pilot studies was found to be the minimal effective dose of landiolol required to normalize the LPS-induced hyperdynamic state in the early stages or hours of sepsis. All rats were killed by Nembutal (sodium pentobarbital, IP, $80 \mathrm{mg} / \mathrm{kg}$ body weight) at $3 \mathrm{~h}$ after LPS or vehicle only. The blood samples were collected from a polyproprene tube catheter inserted into the left carotid artery for blood gas analysis, and hepatic tissues were harvested gently, snap-frozen in liquid nitrogen, and stored at $-80^{\circ} \mathrm{C}$. All animals received humane care and the experimental procedures were approved by the Animal Care and Use Committee of University of Tsukuba.

\section{Measurements of hemodynamic parameters}

The rats were anesthetized with isoflurane inhalation $(1.5 \%, 1 \mathrm{~L} /$ min) and a microtip pressure transducer catheter (SPC-320, Millar Instruments, Houston, TX, USA) was inserted into the left carotid artery $[14,16,17]$. Then arterial blood pressure and heart rate were monitored with a pressure transducer (model SCK-590, Gould, Ohio, USA) and recorded with the use of a polygraph system (amplifier, AP601G, Nihon Kohden, Tokyo, Japan; tachometer, AT-601G, Nihon Kohden; and thermal-pen recorder, WT-687G, Nihon Kohden) $[14,16,17]$.

\section{Measurements of liver injury markers}

Serum aspartate aminotransferase (AST) and alanine aminotransferase (ALT) were measured using kits from Wako Pure Chemical Industries, LTD (Osaka, Japan).

\section{Histopathologic examination}

For histopathology, the hepatic tissue specimens were fixed in $4 \%$ buffered formalin solution, dehydrated through an ethanol series, embedded in paraffin, and sliced into $5-\mu \mathrm{m}$-thick sections. After deparaffinization, sections were stained with hematoxylin and eosin (H\&E) using the standard method. The slides were analyzed by two pathologists in double-blinded fashion. These pathologists have specialization on liver morphology and are affiliated with University of Tsukuba Hospital, Tsukuba, Japan. Histomorphometry was done using light microscopy. The points of acute liver injury are hepatic necrosis, degenerative change, inflammatory cell infiltration, Kupffer cell, hemorrhage, councilman body, sinusoid dilation. These points of acute liver injury score were determined 0 (none: -), 1(mild: \pm ), 2 (moderate: + ), 3 (severe: ++ ), 4 (very severe: +++ ) by a pathologist.

\section{Enzyme immunoassay for TNF- $\alpha$ and ET-1}

The concentration of each respective protein/peptide of interest in plasma/serum and liver tissue extracts was determined using the following kits: serum/plasma and liver levels of TNF- $\alpha$ and ET-1 (R \& D Systems, Minneapolis, MN), according to the manufacturer's protocol.

\section{Statistical Analysis}

The results were expressed as mean $\pm \mathrm{SE}$, and the means were compared by a one-way factorial analysis of variance, followed by Scheffé's test for multiple comparisons. Differences were considered significant at $\mathrm{p}<0.05$.

\section{Results}

Table 1 demonstrates hemodynamic, echocardiogram, and blood gas analysis parameters in experimental animals. Both the systolic and diastolic blood pressure levels were significantly lower at $3 \mathrm{~h}$ after LPS administration and were unaffected following treatment of rats with landiolol for $3 \mathrm{~h}$ (Table 1). Heart rate was significantly increased in LPS group compared to the control group and significantly decreased in LPS-administered rats treated with landiolol (Table 1). Arterial $\mathrm{PaO}_{2}$ was found to be significantly reduced in LPS-administered rats. However, following landiolol administration, there was a significant reversal effect on arterial $\mathrm{PaO}_{2}$ (Table 1). Blood lactate concentrations were increased dramatically after LPS was given and partly normalized with the treatment of landiolol (Table 1).

Representative images from HE staining with the quantitation of liver injury severity by injury score are shown in Figure 1 from the experimental groups studied in the present study. Control group showed normal liver morphology. The morphology of liver from LPSadministered rats at $3 \mathrm{~h}$ showed marked morphological disruptions, such as necrosis $( \pm \sim+)$, degenerative change $(++\sim+++)$, inflammatory cell infiltration $( \pm \sim+)$, Kupffer cell hyperplasia $( \pm \sim+)$, hemorrhage $(+)$, councilman body $( \pm \sim++)$ and sinusoid dilation $( \pm \sim+)$. These histopathological changes were significant in LPS-administered rats compared to those of the control liver group. After three hours treatment with landiolol of LPS-administered rats, there were less pronounced histomorphological disruptions, such as degenerative change $( \pm \sim+)$, inflammatory cell infiltration $(-\sim \pm)$, and hemorrhage $(-\sim+)$ in liver. In addition, there was no remarkable necrosis in liver

\begin{tabular}{|c|c|c|c|}
\hline & Control & LPS & LPS + Landiolol \\
\hline $\mathrm{pH}$ & $7.412 \pm 0.003$ & $7.420 \pm 0.020$ & $7.400 \pm 0.015$ \\
\hline $\mathrm{PaO}_{2}$, torr & $102.6 \pm 2.3$ & $86.5 \pm 2.6^{*}$ & $98.3 \pm 2.6^{\#}$ \\
\hline $\mathrm{PaCO}_{2}$, torr & $39.8 \pm 1.2$ & $34.1 \pm 1.4$ & $32.1 \pm 2.6$ \\
\hline $\mathrm{BE}, \mathrm{mmol} / \mathrm{l}$ & $1.0 \pm 0.6$ & $-3.2 \pm 0.5^{*}$ & $-5.2 \pm 1.0^{*}$ \\
\hline $\mathrm{Lac}, \mathrm{mmol} / \mathrm{l}$ & $0.8 \pm 0.2$ & $3.1 \pm 0.4^{*}$ & $1.6 \pm 0.1^{\#}$ \\
\hline $\mathrm{HCO}_{3}, \mathrm{mmol} / \mathrm{l}$ & $24.8 \pm 0.8$ & $21.5 \pm 0.6$ & $20.0 \pm 1.2^{*}$ \\
\hline Heart Rate, bpm & $462.0 \pm 7.7$ & $497.0 \pm 4.9^{*}$ & $465.0 \pm 8.8^{\#}$ \\
\hline Systolic BP, $\mathrm{mmHg}$ & $118.0 \pm 0.5$ & $102.0 \pm 4.4^{*}$ & $101.0 \pm 4.5^{*}$ \\
\hline Diastolic BP, $\mathrm{mmHg}$ & $77.0 \pm 2.0$ & $66.0 \pm 1.2^{*}$ & $65.0 \pm 1.1^{*}$ \\
\hline
\end{tabular}

LPS, lipopolysaccharide; BE, base excess; Lac, lactate; BP, blood pressure Data are mean \pm S.E.

${ }^{*} \mathrm{p}<0.05$ vs control, ${ }^{\#} \mathrm{p}<0.05$ vs LPS

Table 1: Hemodynamic and blood gas analysis parameters in experimental animals. 
A

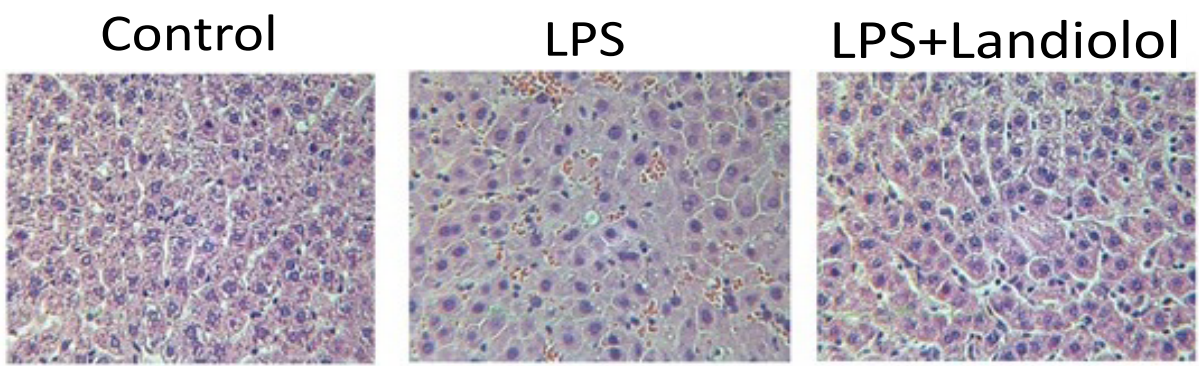

$\mathrm{B}$

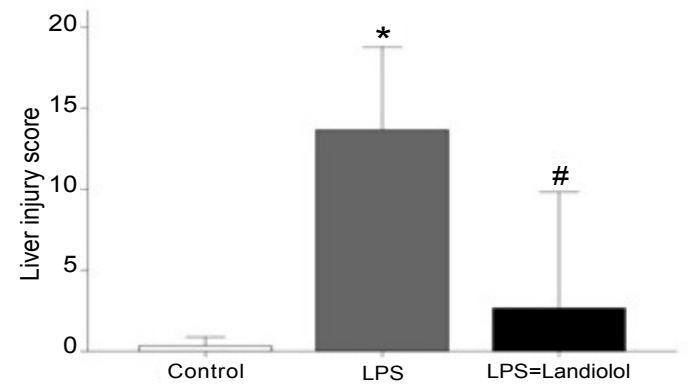

Figure 1: Morphological findings by hematoxylin and eosin staining in the liver tissues in control rats, $3 \mathrm{~h}$ after lipopolysaccharide (LPS) administration, and $3 \mathrm{~h}$ after LPS plus landiolol hydrochloride administration; (A): representative images; (B) summary graph of injury score. Magnification, 100X.
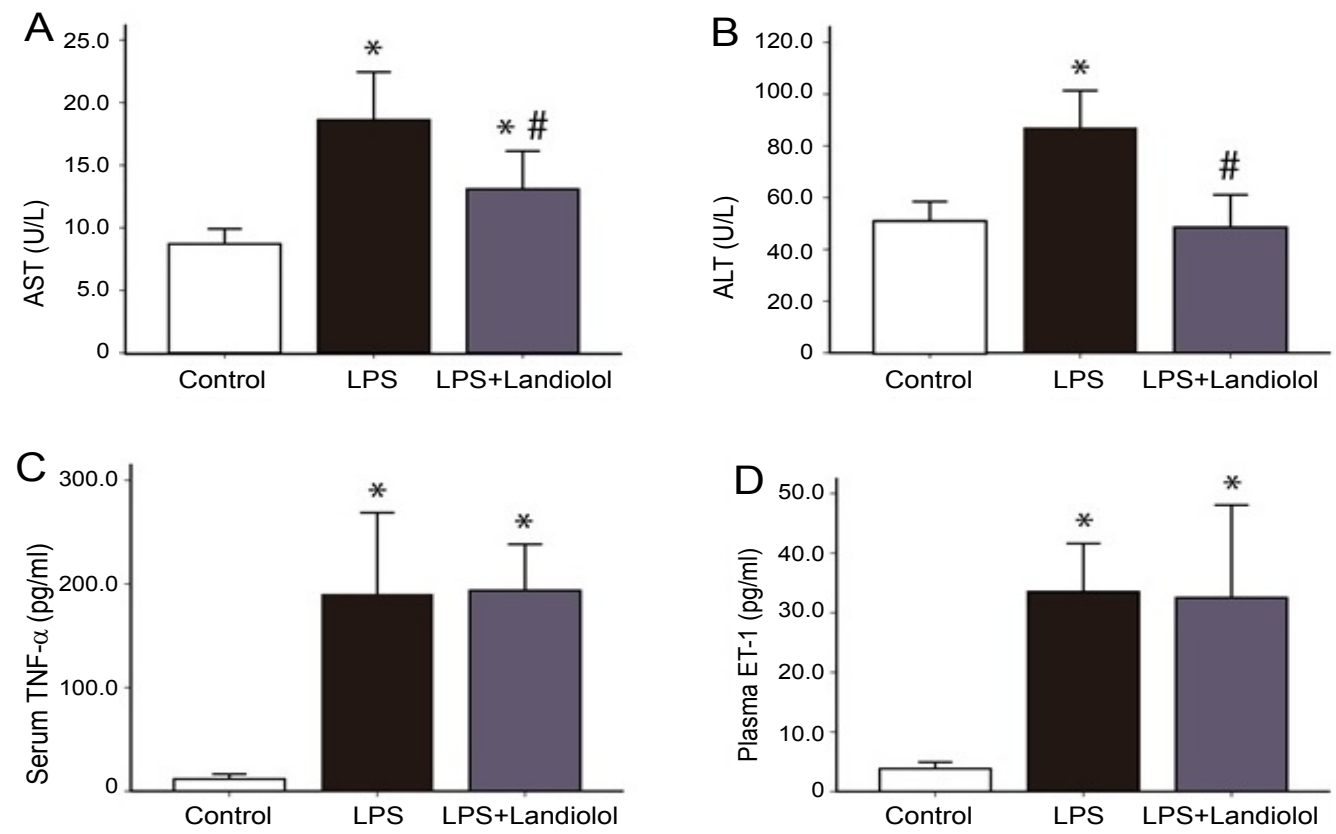

Figure 2: Serum AST (A), serum ALT (B), serum TNF- $\alpha$ (C) and plasma ET-1 (D) levels of the control, $3 \mathrm{~h} \mathrm{LPS-administered} \mathrm{rats,} \mathrm{and} 3 \mathrm{~h}$ landiolol-treated LPSadministered rats. Values are mean $\pm S E{ }^{*} p<0.05$ vs. control; $\# p<0.05$ vs. LPS.

tissue in septic rats after landiolol treatment. As shown in Figure 2B, the enhanced injury score in LPS-administered rats were significantly ameliorated following treatment of rats with landiolol for $3 \mathrm{~h}$.

Levels of serum AST and ALT (Figure 2A and 2B), increased significantly at $3 \mathrm{~h}$ after LPS administration, when compared to the control rats and the rise biochemical markers of interest were significantly ameliorated following the treatment of LPS-administered rats with landiolol. The serum levels of TNF- $\alpha$ were elevated after administration of LPS (Figure 2C). However, landiolol treatment failed to normalize the elevated serum levels of TNF- $\alpha$ in sepsis rats. Consistent with our previous report [16,17], the present data show elevated levels of plasma ET-1 in sepsis rats (Figure 2D), which was unaffected by 
landiolol at $3 \mathrm{~h}$ post-treatment. Protein expression levels of TNF- $\alpha$ were elevated in liver tissue after LPS administration, compared to control group, and landiolol treatment significantly normalized the elevated hepatic levels of TNF- $\alpha$ (Figure 3A). In liver tissue, ET-1 peptide levels were significantly higher in LPS-administered group compared to the control group. However, landiolol treatment did not alter levels of enhanced ET-1 (Figure 3B) in septic rat liver tissues.

\section{Discussion}

The key findings of the present study are that: 1) treatment of septic rats with landiolol $3 \mathrm{~h}$-post administration was effective in ameliorating the histopathological and biochemical (AST and ALT) parameters of liver injury, respectively; 2) this improvement in the condition of liver injury in LPS-administered rats was accompanied by significant normalization of hepatic TNF- $\alpha$ levels; 3 ) finally the amelioration of liver injury by landiolol treatment, as observed in the present study, may not involve ET-1 levels in liver tissue in septic rats.

Landiolol hydrochloride is an intravenously administered, ultra short-acting $\beta 1$-blocker with an elimination half-life of 3-4 min and $\approx 8$-fold greater cardioselectivity than esmolol in vitro [1821]. It is approved in Japan for the treatment of intraoperative and postoperative tachyarrhythmias, but is also used in clinical practice to prevent postoperative tachyarrhythmias, such as atrial fibrillation after coronary artery bypass grafting [18-21]. As an ultra short-acting $\beta 1$ blocker with a rapid onset of action and readily titratable and rapidly reversible effects, landiolol represents an important agent for the management of intraoperative and postoperative tachyarrhythmias
[18-21]. Very recently, landiolol has been reported to confer organ protection in sepsis animal models through various pathways [15-17]. Recent studies have demonstrated that co-treatment of landiolol in LPS-induced systemic inflammation protects against acute lung injury and cardiac dysfunction in a rat inflammation models $[15,16]$ through the suppression of inflammatory markers. Consistent with these previous findings, the current study also found that landiolol treatment confers hepatic protection both at histomorphological as well as biochemical levels during the early hours of sepsis. Proinflammatory mediators, such as LPS and TNF- $\alpha$, can directly lead to hepatocellular damage [22]. It has been shown in a previous study that LPS and TNF- $\alpha$ may play a central role in the development of acute hepatic failure after severe trauma and sepsis by directly or indirectly inducing hepatocyte necrosis rather than apoptosis [22]. In the current study, we found increased levels of TNF- $\alpha$ both at circulatory and hepatic tissue level after sepsis induction through LPS administration. This upregulation can directly lead to hepatocellular damage.

Amelioration of liver injury by landiolol may involve the inhibitory action of landiolol on local elevated levels of TNF- $\alpha$ in liver tissue of LPS-induced sepsis in rats. In the present study, we found attenuation of histomorphological hepatic changes, such as degeneration, inflammatory infiltration and hemorrhage following co-treatment of LPS with landiolol by three hours. Interestingly, while landiolol significantly normalized the local elevated levels of TNF- $\alpha$ in the livers of LPS-induced sepsis in rats, it (landiolol) failed to reverse increased levels of TNF- $\alpha$ at the circulatory level. For the moment, we do not have any specific explanation underlying the differential effects of
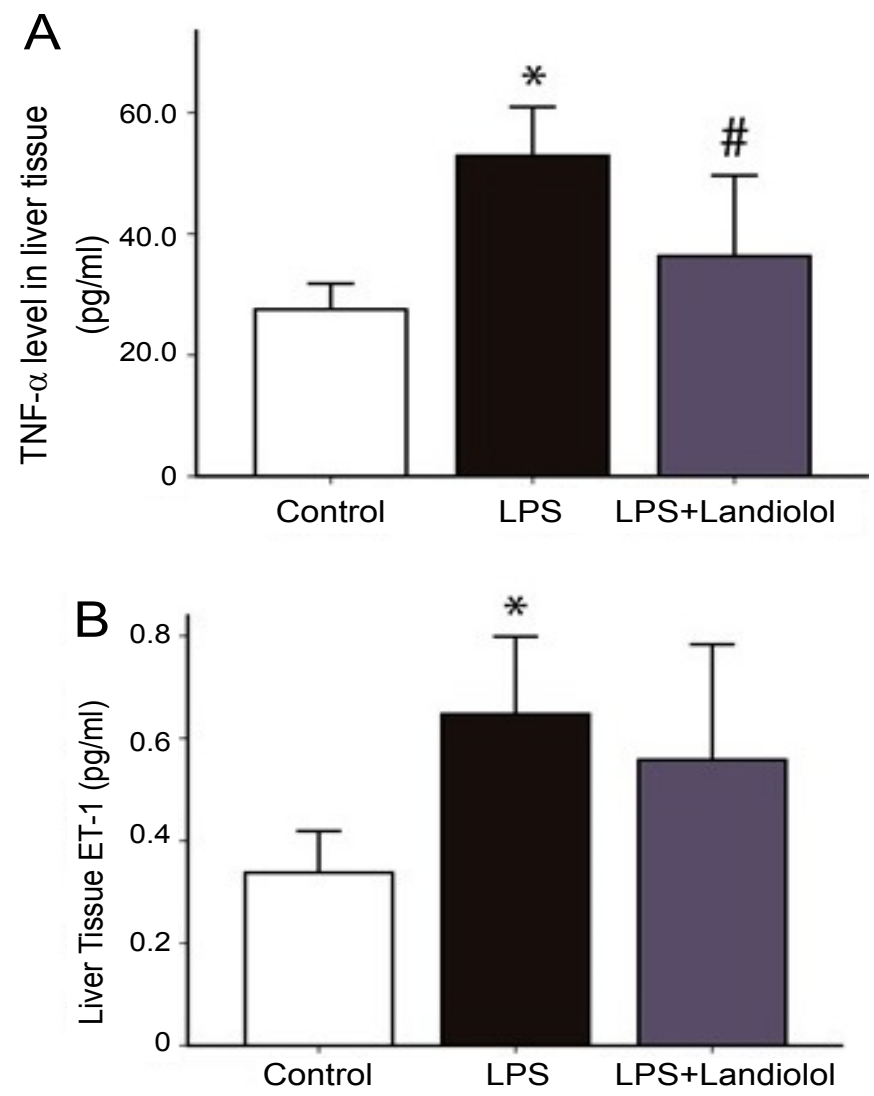

Figure 3: Protein expression levels of TNF- $\alpha(A)$ and ET-1 (B) peptide levels in liver tissues of the control group, LPS-administered rats (3 h-post treatment), and landiolol-treated LPS-administered rats ( $3 \mathrm{~h}$-post treatment). These data were generated by ELIZA. Values are mean \pm SE ${ }^{*} p<0.05$ vs. control; \#p<0.05 vs. LPS 
Citation: Yoshino Y, Jesmin S, Islam M, Shimojo N, Sakuramoto H, et al. (2015) Landiolol Hydrochloride Ameliorates Liver Injury in a Rat Sepsis Model by Down Regulating Hepatic TNF-A. J Vasc Med Surg 3: 194. doi:10.4172/2329-6925.1000194

landiolol on TNF- $\alpha$ expression in liver versus circulation in septic rats. Further studies are required to clarify these issues. ET-1, a potent vasoconstrictor, has been implicated in the pathogenesis of sepsis. Plasma levels of ET-1 have been shown to be significantly higher in septic patients [11] and their levels (ET-1) have a clear correlation with morbidity and mortality in septic patients, suggesting an involvement of ET in the pathogenesis of septic shock in humans [12]. Further, it has been suggested that ET contributes to the dysfunction of several vital organ systems during septic shock. In our previous study, we have shown that ET-1 is highly upregulated in hepatic tissues in septic rats [14]. In fact, ET-1 is a powerful vasoactive peptide and is secreted by various cells in liver including vascular and sinusoidal endothelial cells and hepatic stellate cells $[23,24]$.ET- 1 also causes strong vasoconstrictive action after binding to its receptor [25]. Thus, a growing body of evidences suggests a potential role of ET-1 in the pathogenesis of liver injury. The present findings show that the amelioration of liver injury in sepsis by landiolol does not involve ET-1 pathway, contrary to our recent studies that demonstrated the significant reversal of landiolol on elevated cardiac and renal tissues ET-1 signaling system in sepsis. In contrast, in the current study, landiolol treatment did not normalize upregulated ET-1 levels in liver tissues of septic rats. Thus, it can be concluded that landiolol has organ-specific (reversal) effects on altered ET-1 levels during sepsis. The present study has several limitations. We cannot rule out from the present findings that what types of liver cells are significantly impacted by the landiolol treatment in sepsis. In vitro studies using various cell types of liver should be conducted in future. In addition, other inflammatory cytokines should be investigated in current study design in depth. Longer duration treatment with landiolol in sepsis models can be done in future in current experimental setting.

\section{Conclusion}

The present study demonstrates that landiolol hydrochloride, a selective ultra-short acting beta blocker, ameliorates liver injury in a rat model of sepsis possibly through the suppression of elevated hepatic TNF-a levels.

\section{Acknowledgements}

This work was supported in part by a Grant-in-Aid for Scientific Research B and $C$ from the Ministry of Education, Culture, Sports, Science and Technology of Japan (22390334, 23592025, 23406037, 23406016, 23406029, 24406026, 25462812 and 25305034) and Japan Society for the Promotion of Science.

\section{References}

1. Singh S, Evans TW (2006) Organ dysfunction during sepsis. Intensive Care Med 32: 349-360.

2. Korish AA, Arafa MM (2011) Propolis derivatives inhibit the systemic inflammatory response and protect hepatic and neuronal cells in acute septic shock. The Brazilian journal of infectious diseases. The Brazilian Society of Infectious Diseases 15: 332-338

3. Hozumi H, Tada R, Murakami T, Adachi Y, Ohno N (2013) Comparative analysis of hepatic CD14 expression between two different endotoxin shock model mice: relation between hepatic injury and CD14 expression. PloS one 8: 53692

4. Marzocco S, Di Paola R, Ribecco MT, Sorrentino R, Domenico B, et al. (2004) Effect of methylguanidine in a model of septic shock induced by LPS. Free Radic Res 38: 1143-1153.

5. Szabó C, Módis K (2010) Pathophysiological roles of peroxynitrite in circulatory shock. Shock 34 Suppl 1: 4-14
6. González-Terán B, Cortés JR, Manieri E, Matesanz N, Verdugo Á, et al. (2013) Eukaryotic elongation factor 2 controls TNF-Ît translation in LPS-induced hepatitis. J Clin Invest 123: 164-178.

7. Dhainaut JF, Marin N, Mignon A, Vinsonneau C (2001) Hepatic response to sepsis: interaction between coagulation and inflammatory processes. Crit Care Med 29: S42-47.

8. Pastor CM, Billiar TR, Losser MR, Payen DM (1995) Liver injury during sepsis. J Crit Care 10: 183-197.

9. Luster MI, Germolec DR, Yoshida T, Kayama F, Thompson M (1994) Endotoxininduced cytokine gene expression and excretion in the liver. Hepatology 19 480-488.

10. Doi F, Goya T, Torisu M (1993) Potential role of hepatic macrophages in neutrophil-mediated liver injury in rats with sepsis. Hepatology 17: 1086-1094.

11. Battistini B, Forget M A, Laight D (1996) Potential roles for endothelins in systemic inflammatory response syndrome with a particular relationship to cytokines. Shock 5: 167-183

12. Pittet JF, Morel DR, Hemsen A Gunning K, Lacroix JS, et al. (1991) Elevated plasma endothelin- 1 concentrations are associated with the severity of illness in patients with sepsis. Ann. Surg 213: 261-264

13. Weitzberg E, Lundberg JM, Rudehill A (1991) Elevated plasma levels of endothelin in patients with sepsis syndrome. Circ Shock 33: 222-227.

14. Zaedi S, Jesmin S, Yamaguchi N, Shimojo N, Maeda S, et al. (2006) Altered expression of endothelin, vascular endothelial growth factor, and its receptor in hepatic tissue in endotoxemic rat. Exp Biol Med (Maywood) 231: 1182-1186.

15. Hagiwara S, Iwasaka H, Maeda H, Noguchi T (2009) Landiolol, an ultrashortacting beta1-adrenoceptor antagonist, has protective effects in an LPS-induced systemic inflammation model. Shock 31: 515-520.

16. Seki Y, Jesmin S, Shimojo N, Islam MM, Rahman MA, et al. (2014) Significant reversal of cardiac upregulated endothelin-1 system in a rat model of sepsis by landiolol hydrochloride. Life Sci 118: 357-363.

17. Ogura Y, Jesmin S, Yamaguchi N, Oki M, Shimojo N, et al (2014) Potentia amelioration of upregulated renal HIF-1alpha-endothelin-1 system by landiolol hydrochloride in a rat model of endotoxemia. Life Sci 118: 347-356.

18. Plosker GL (2013) Landiolol: a review of its use in intraoperative and postoperative tachyarrhythmias. Drugs 73: 959-977.

19. Iguchi S, Iwamura H, Nishizaki M, Hayashi A, Senokuchi K, et al. (1992) Development of a highly cardioselective ultra short-acting beta-blocker, ONO1101. Chem Pharm Bull (Tokyo) 40: 1462-1469.

20. Momomura S (2013) Acute rate control in atrial fibrillation with left ventricular dysfunction. Circ J 77: 893-894.

21. Sakaguchi M, Sasaki Y, Hirai H, Hosono M, Nakahira A, et al. (2012) Efficacy of Landiolol Hydrochloride for Prevention of Atrial Fibrillation After Heart Valve Surgery. International heart journal 53: 359-363

22. Wang JH, Redmond HP, Watson RW, Bouchier-Hayes D (1995) Role of lipopolysaccharide and tumor necrosis factor-alpha in induction of hepatocyte necrosis. Am J Physiol 269: G297-304

23. Rockey DC, Fouassier L, Chung JJ, Carayon A, Vallee, P, et al. (1998) Cellula localization of endothelin-1 and increased production in liver injury in the rat: Potential for autocrine and paracrine effects on stellate cells. Hepatology 27 $472-480$.

24. Pinzani M, Milani S, Defranco R, Grappone C, Caligiuri A, et al. (1996) Endothelin 1 is overexpressed in human cirrhotic liver and exerts multiple effects on activated hepatic stellate cells. Gastroenterology 110: 534-548.

25. Okumura S, Takei Y, Kawano S, Nagano K, Masuda E, et al. (1994) Vasoactive effect of endothelin-1 on rat liver in vivo. Hepatology 19: 155-161. 